\title{
STABILIZATION OF THE NUMERICAL PRIMING TO STABILIZATION PHENOMENON IN THE CRUSHING OF A COMPOSITE PANEL USING LOCAL AND DIFFUSE DAMAGE LAWS
}

\author{
$\underline{\text { Christine Espinosa }}^{1 *}$, Floran Tostain ${ }^{1}$, Samuel Rivallant $^{1}$ \\ ${ }^{1}$ Université de Toulouse - ISAE-SUPAERO - ICA/MS2M-MSC UMR CNRS 5312 \\ 3 Rue Caroline Aigle - 31400 Toulouse - France \\ e-mail: Christine.Espinosa@isae-supaero.fr*;
}

Floran.Tostain@isae.fr; $\underline{\text { Samuel.Rivallant@isae-supaero.fr }}$

Keywords: Composite, Carbon Fibers, Crush, Crash, Edge Compression, Damage, Fragmentation, Splaying, Debris, Energy Absorption.

\begin{abstract}
This study lies within the general scope of damage and rupture prediction during transverse crash (edge crushing) of a beveled composite plate using numerical simulation. Structures under consideration are aeronautical composite structures in purpose of design and certification. The present study proposes as an enhancement of an existing crushing model the introduction of a continuous softening law to represent the highly complex local fragmentation process at the vicinity of the crushing process on the ground. It is shown how the added softening stress strain law helps the numerical stability and the reproduction of all the processes in the different phases of the crushing process.
\end{abstract}




\section{INTRODUCTION}

This work is devoted to the analysis of the link between the initiation phase of the crushing of a chamfered composite plate up to the peak force, and the stability of the crushing process in a steady phase of the process of energy absorption. The analysis aims to evaluate the effect of interlaminar strength resistance and the predominant ruin modes corresponding to different combinations of strength values. More precisely, we propose a strategy of modelling the structure that uses two behavior laws, in order to represent both the creation of new surfaces by delamination and rupture through plies induced by diffuse damage, and delamination induced by specific dust and chip formation at the vicinity to the ground. We are concerned here with CFRP composite plates crushed at about $9 \mathrm{~m} / \mathrm{s}$, loading a heavy carriage at the ear face to represent the inertial effects of the structure to protect. Numerical simulations are compared with experimental results.

\subsection{General purpose and position of the work}

The Specific Energy Absorption (SEA) is the characteristics of a structure that represents its scalar capacity to absorb the of energy during either quasi-static or dynamic crushing processes. The SEA is defined as the ratio between the mean crushing force $F_{\text {moy }}$ by the specific mass of matter that is involved and in the crushing process. This mass is obtained by the product of the specific mass of the material $\rho_{\mathrm{s}}$ and the involved area $A_{s}$. The energy is given by (1):

$$
S E A=\frac{F_{\text {moy }}}{\rho_{S} \times A_{S}}
$$
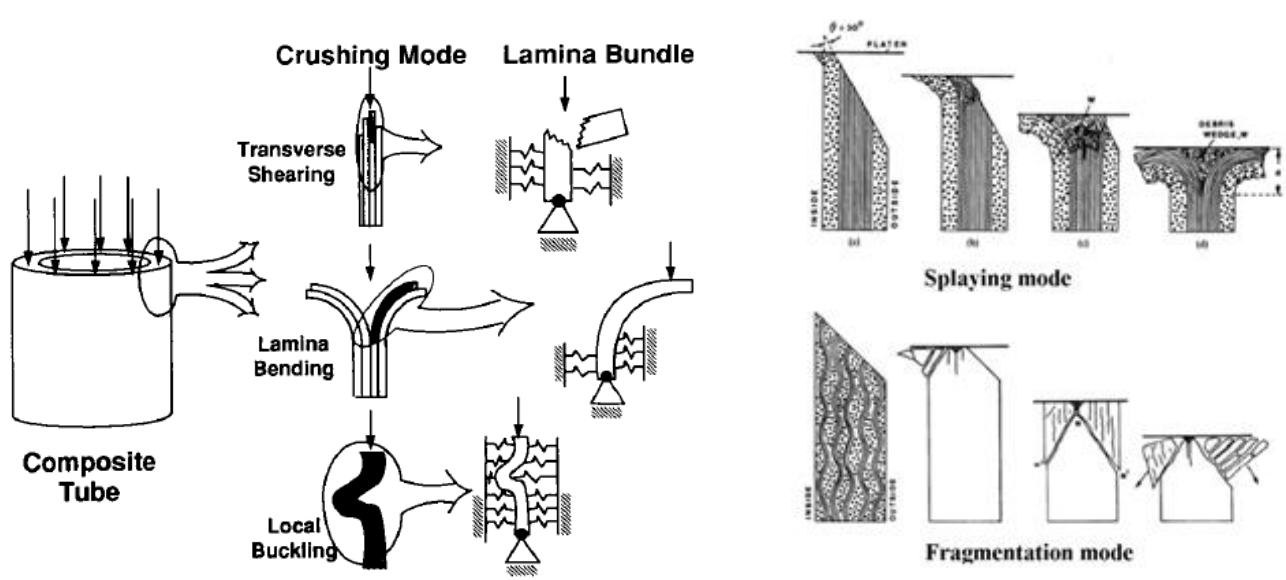

Figure 1: Left: Crushing modes of a composite tube [1]; Right: splaying and fragmentation crushing modes for chamfered plates [2]

The process is controlled by the ruin modes of the material, and constrained by the structure geometry. The SEA is conditioned by the co-existence and the competition of both ruin modes. The material ruin modes (fiber breakage, fiber/matrix decohesion, matrix cracking) can furthermore depend upon strain rate effects and distributed defects which are responsible of uncertainty on their relative contribution. The structure ruin modes are mainly described by geometrical aspects of the contact surface to the ground, and of the integrity (buckling, delamination and lamina bending, lamina bundle). Theses modes are illustrated on Figure 1. In particular, the open or closed form of the structure is a crucial point $[1,2]$. 
For certification to crash of new generations of aircraft structures without landing gears, it is necessary to design energy-absorbing structures that are linked to the fuselage both maximizing the absorbed energy and minimizing the total mass $[3,4]$. The "quality" of the optimum design of energy absorption structure in driven by two criteria: the structure should stay in a stable energy absorption process, and a maximum volume of material should be damaged during this process. The design must take into account the complexity of loading conditions and the competition of structural long-dimensions behaviours with the local structural thicknessdimensions damages [5, 7]. Even if efforts have been made to represent material properties degradations, debris wedge generation, and residual shape of structures, initiation is the most critical phase $[2,7,8]$ and the part of the process where fragmentation is the more active.

Understanding the interactions between the structural geometry evolution and the degradation of the material properties is crucial. Well known for metallic materials, it is still a challenge to determine for composite materials for which the link between initiation in the first beginning of the crushing process up to the peak force, and stabilization of the process to reach a 'stable' or 'steady' crushing force. It is the main interest of this study to contribute to this understanding, and to propose a numerical model that controls all these phenomena.

\subsection{Structure effects on initiation and stabilization of the process}

Structural effects are associated to boundary evolutions, since boundaries define the geometries either of the whole structure or the assemble of substructures. It is proposed here to define essentially two categories of boundaries for the crash/crush absorbers. First, boundaries through which the compression is applied (extremities), in particular the boundary which is in contact with the ground/wall which represent indeed the obstacle to the structure global displacement. This boundary is responsible of the load increase, and is the place where the internal energy is released through decohesions of internal link, and where the three modes of energy absorption appear. Boundaries at the vicinity of the obstacle evolve as failures and ruptures appear and fragments are evacuated all the process long. Second, boundaries where internal energy is not increased but is only released because the fragments or parts separate from each other. The resulting SEA describes the balance between these entering and exiting energies through the evolution of the boundaries, or solely exiting energies through the second kind. Following this boundaries decomposition, it is proposed to analyze and model separately the phenomena appearing at the vicinity of the obstacle, and those appearing in the rest of the structure.

Studies of the literature devoted to phenomena at the vicinity of the obstacle are much more numerous for closed profiles because these structures are historically used by transportation companies (cars for example). For closed structures it is interesting to focus on the effects of contact shape and geometry between the structure and the obstacle on the mean crushing force and stabilization of the crushing process. Interesting works can be found using variable thickness in the structure height (distance to the obstacle) to increase progressively the contact surface, thus avoiding any loss of contact or dynamic buckling or bundling effects [9-11]. The shape of the structure (polygonal) and thus of the contact also has an effect on the number of fragments and chips and on the global structure strength [12]. Some profiles are demonstrated to activate more progressive tear related by the authors to the stable distribution of the contact force between the rigid obstacle and the structure, for different composite structures [12, 3-4]. It is interesting to link this observation with the effect of the contact angle $\left(20^{\circ}\right)$ on the sudden and catastrophic breakdown of the structure for unidirectional CFRP [13], which is not the case for pultruded composite [14]. Pultruded composites appear to be more stable and the 
structural effects are much more similar to metallic structure for which the shape of the initiator as a low effect on the energy absorption performance [15]. As far as we are concerned, for unidirectional CFRP laminates, it has been proved that the trigger and the angles of contact have a great effect on the initiation of the crushing process. Furthermore, the mean crushing force depends considerably to what happens in the few instants of the contact between the chamfered structure and the obstacle [4, 6, 16-17]. This influence is due to the kind of material and the evolution of the contact conditions between the structure boundaries and the obstacle [18-19]. As a consequence, it is of primary importance to represent the specificity of the material behavior and the contact non-linearities between all the boundaries as they are more and more created forward and interacting with the obstacle and the created fragments. This is the object of the first part of this work.

\subsection{Material effects on initiation and stabilization of the process}

In parallel with these local effects, it can be noticed that drops down and increase of resisting forces after the peak force and in the mean force plateau, are conditioned by the capacity of the plies to continuously transmit the loading in the height of the structure (distance to the obstacle) or to break the loading path through local buckling kink band formation and ruptures (confined plies) or outward global buckling of plies [20]. This phenomenon has been identified by Lavoie [21] whose fundamental work shows that the plateau value and the oscillations around the mean value for APC2 laminates is twice that of CFRP or GR-Kv epoxy laminates. Indeed, the PEEK matrix exhibits a high interlaminar fracture toughness which is suspected to be at the origin of these observations. The link between the frequency of the mean force oscillations in the plateau region and the width of the kink band can be due to the relative value of the interlaminar strength and the longitudinal strength and critical value of strength in compression of laminates [22]. The more the fiber proportion, the higher the SEA and the amplitude of the mean force in the plateau region [20]. As for the case of the influence of the structure profile, these result must be put into perspective with the kind of fibers [23]. Nevertheless, it is proven that the stability of the crushing process in the plateau region, and the amplitude and frequency of the mean force oscillations, are both related to damage modes of the material in the core of the laminate but are also influenced by the waves of loading that come directly from the contact vicinity. This loading transfer is continuous up to the limit load of the material. It is thus crucial to represent in a proper way the damage modes and the relative strength in the core and between the plies of the laminate. This interaction of the interlaminar strength and the pies damage is the point of interest of the second part of this work.

\section{COMPOSITE LAMINATE CRUSHING PROCESS: MODELLING STRATEGY}

Based on observations made from experimental testing campaigns, different mechanical models were proposed in previous studies [8, 16-17]. The basic model used in this work is the nonlinear elastic anisotropic homogenized continuum damage mechanics model developed in the frame of the irreversible thermodynamics [17]. In order to circumvent the problems coming from the local compression at the contact on the ground, the elements that face the ground are changed the material model into the MCS and FFC models to follow the strategy proposed by [16]. These elements are eroded when the maximum load bearing criterion is reached. This simulation strategy is illustrated on Figure 2. Damage is represented at the mesoscale (ply size) which is the scale under interest. 


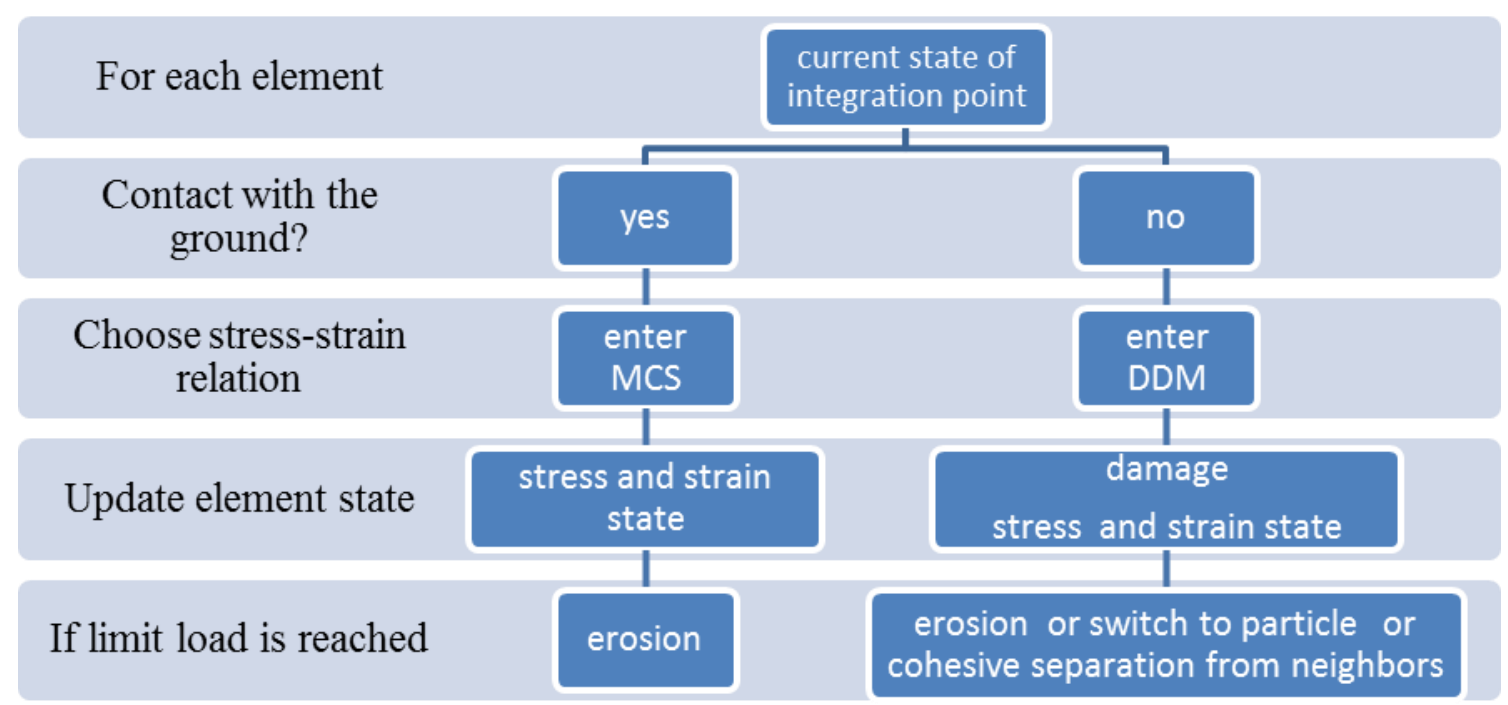

Figure 2: Deep ply damage and localized fragmentations models switch.

The continuous diffuse damage model is available for 3D material, whatever the fibre orientations are, and can manage strain rate effects (not considered here, despite the observation of Lavoie [21]). Modes of ruin are represented by criteria obtained from experiments and are directly related to the deformation energy. The MCS and FFC models have been characterized by other experiments.

\subsection{Continuous diffuse damage model}

The continuous Diffuse Damage Model has been developed on the basis of the previous work of Ylias [17]. Enhancements have been proposed to adapt the model to the strategy described on Figure 2. The DDM emphasizes that anisotropy is unchanged after damage and the material remains elastic in each ply. Thus, as done "classically", damage is modelled through its effect on the elastic rigidity loss in further loading or unloading without a change in the anisotropy directions until it reaches the value of 1 : di is the damage of $E_{i i}$ for $i=1,3, d 4$ is the damage of $\mathrm{G}_{12}$, d5 of $\mathrm{G}_{23}$ and $\mathrm{d} 6$ of $\mathrm{G}_{13}$.

The model distinguishes 6 damage variables $\{\mathrm{di}\}, \mathrm{i}=1,6$ affecting the stiffness of the ply, and 6 failure modes $\{\mathrm{fj}\}, \mathrm{j}=1,6$ (eq. 2-7), where:

$<$.> defines the Macauley brackets,

$X_{T}$ and $X_{C}$ are respectively tensile/compressive failure stresses in fibres direction,

$Y_{T}$ and $Y_{C}$ are respectively tensile/compressive damage threshold stresses in direction 2,

$Z_{T}$ and $Z_{C}$ are respectively tensile/compressive damage threshold stresses in direction 3 ,

$S_{12}, S_{23}$ and $S_{13}$ are respectively shear damage threshold stresses,

$r_{i} \in[1, \infty]$ is called the limit load ratio.

$$
\begin{gathered}
f_{1}\left(\varepsilon, r_{1}\right)=\left[\frac{E_{1}\left\langle\varepsilon_{1}\right\rangle}{X_{T}}\right]^{2}+\left[\frac{G_{12} \varepsilon_{12}^{2}+G_{13} \varepsilon_{13}^{2}}{S_{f s}^{2}}\right]-r_{1}^{2}=0 \\
f_{2}\left(\varepsilon, r_{2}\right)=\left[\frac{E_{1}\left\langle-2 \varepsilon_{1}\right\rangle+\left\langle-E_{2} \varepsilon_{2}-E_{2} \varepsilon_{3}\right\rangle}{2 X_{C}}\right]^{2}-r_{2}^{2}=0
\end{gathered}
$$

tension fiber direction 


$$
\begin{gathered}
f_{3}\left(\varepsilon, r_{3}\right)=\left[\frac{E_{3}\left\langle-\varepsilon_{3}\right\rangle}{Z_{C}}\right]^{2}-r_{3}^{2}=0 \\
f_{4}\left(\varepsilon, r_{4}\right)=\left[\frac{E_{2}\left\langle-\varepsilon_{2}\right\rangle}{Y_{C}}\right]^{2}-r_{4}^{2}=0 \\
f_{5}\left(\varepsilon, r_{5}\right)=\left[\frac{E_{2}\left\langle\varepsilon_{2}\right\rangle}{Y_{T}}\right]^{2}+\left[\frac{G_{12} \varepsilon_{12}}{S_{12^{\circ}}+S_{S R B}}\right]^{2}+\left[\frac{G_{23} \varepsilon_{23}}{S_{23^{\circ}}+S_{S R B}}\right]^{2}-r_{5}^{2}=0 \\
f_{6}\left(\varepsilon, r_{6}\right)=\left[\frac{E_{3}\left\langle\varepsilon_{3}\right\rangle}{Z_{T}}\right]^{2}+\left[\frac{G_{13} \varepsilon_{13}}{S_{13^{\circ}}+S_{S R B}}\right]^{2}+\left[\frac{G_{23} \varepsilon_{23}}{S_{23^{\circ}}+S_{S R B}}\right]^{2}-r_{6}^{2}=0 \quad \text { matrix cracking }
\end{gathered}
$$

At the undamaged state, limit load ratios ri are set to 1 . They evolve following the current state of damage using the first thermodynamics principle and following (eq. 8) [15]:

$$
\dot{d}_{i}(\sigma, \omega, \dot{\varepsilon})=\sum_{j=1}^{5} q_{i j} \dot{\phi}_{j}\left(m, r_{j}\right), \quad \phi_{j}=1-e^{\frac{1}{m}\left(1-r_{j}^{m}\right)}, \quad r_{j} \geq 1
$$

\subsection{Mean Crushing Stress and Free Face Crushing laws for local fragmentation}

The stress-strain curve of elements at the tip of the plies that are in contact with the ground is similar to an elastic perfectly plastic behaviour in compression (Figure 3).
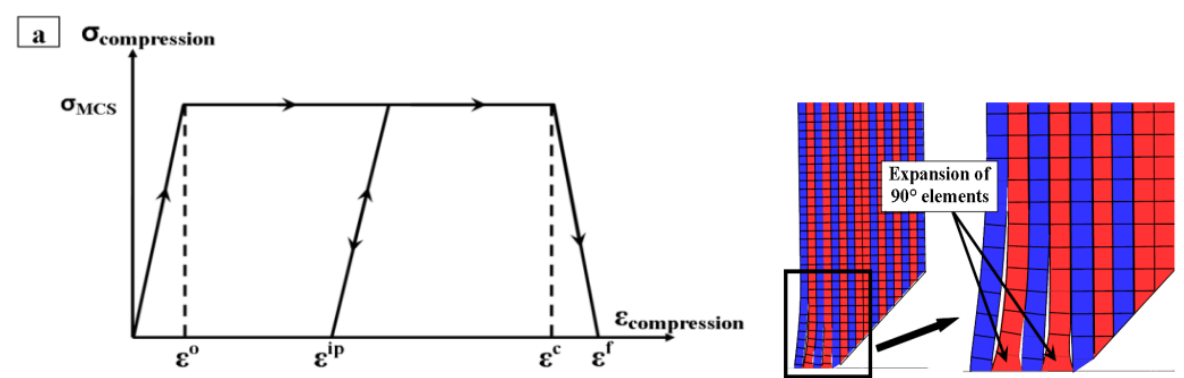

Figure 3: Left: MCS law for both $0^{\circ}$ and $90^{\circ}$ plies; Right: expansion of $90^{\circ}$ elements to allow initiation of delamination using cohesive elements.

Data used in this study have been characterized by Israr \& al. [16]. The elastic deformations $\varepsilon_{0^{\mathrm{p}}}^{0}$ and $\varepsilon_{90^{\mathrm{p}}}^{0}$ are given by equations (9) and (10), where $\mathrm{H}_{11 \mathrm{c}}=101 \mathrm{GPa}$, and $\mathrm{H}_{22 \mathrm{c}}=$ $9 \mathrm{GPa}$. The maximum compression strain $\varepsilon_{\mathrm{c}}$ and failure strain $\varepsilon_{\mathrm{f}}$ are the same for plies at $0^{\circ}$ and $90^{\circ}$ and are respectively $-3(95 \%)$ and $-4.6(99 \%)$.

$$
\begin{aligned}
& \varepsilon_{0^{\mathrm{p}}}^{0}=\frac{\sigma_{M C S 0^{\circ}}}{H 11 c} \text { with } \mathrm{H}_{11 \mathrm{c}}=101 \mathrm{GPa} \text { and } \sigma_{M C S 0^{\circ}}=277 \mathrm{MPa} \\
& \varepsilon_{90^{\circ}}^{0}=\frac{\sigma_{M C S 90^{\circ}}}{H 22 c} \text { with } \mathrm{H}_{22 c}=9 \mathrm{GPa} \text { and } \sigma_{M C S 90^{\circ}}=270 \mathrm{MPa}
\end{aligned}
$$

In order to treat differently the elements where the continuous diffuse damage law is used or the Mean Crushing Sress law is used, and to reduce spurious oscillations in the contact force, the FFC is implemented in a user program and called as a sub-routine in the user material of Impetus Afea. The principle of the Free Face Crushing is illustrated on Figure 4. 

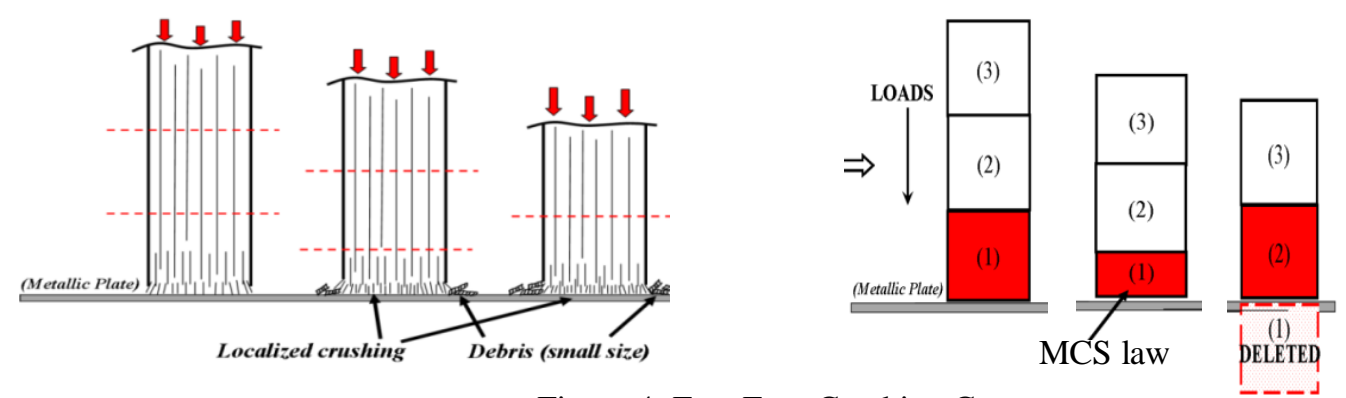

Figure 4: Free Face Crushing Concept.

\subsection{Cohesive failure}

The cohesive failure is generated through a tied contact with failure and propagated using a quadratic failure criterion using mode I and mode II strength related to $\mathrm{G}_{\mathrm{Ic}}$ and $\mathrm{G}_{\mathrm{IIC}}$ energy release rates. Energy release follows a linear tension to opening relationship. In order to limit the mesh size effect in the opening crack propagation, a scale factor $\zeta$ is introduced [17].

\subsection{Experimental case under interest}

$\left[\left(0^{\circ} / 90^{\circ}\right)_{4}\right] \mathrm{s}$ samples are made of $268 \mathrm{~g} / \mathrm{m}^{2}$ T700GC/M21e prepreg (35\% of resin), so that the global density is $1535 \mathrm{~kg} / \mathrm{m}^{3}$. Sample plates are $160 \mathrm{~mm}\left(0^{\circ}\right.$ _fiber_direction $) \times 60 \mathrm{~mm}$ (90 ${ }^{\circ}$ _perp.plan_direction) chamfered rectangular plates so that initiation is conditioned by the wedge angle, here $45^{\circ}$. Each ply is $0,25 \mathrm{~mm}$ thick. A heavy carriage is fixed at the upper boundary of the plate to adjust the desired initial kinetic energy. The mass chosen here is $36 \mathrm{~kg}$, and the initial velocity is $9 \mathrm{~m} / \mathrm{s}$. The free height for crushing is $20 \mathrm{~mm}$. The experimental setup is described in detail in references $[7-8,16,17]$.

\subsection{Models inputs}

The finite element model is described Figure 5 (shortened in order to show details; the real model has the same height as in the experiment). Finite elements for the composite structure are eight nodes degree 1 fully integrated isogeometric elements, with an edge length of $0,25 \mathrm{~mm}$. To generate the chamfer, lines of elements in the stacking plies are 1 element shorter from top to bottom along the (z) direction of the frame. In order to stabilize the ignition of the crushing process, the mesh of the extremity of the plate and the contact parameters has been smoothed to get a strait surface for the chamfer.
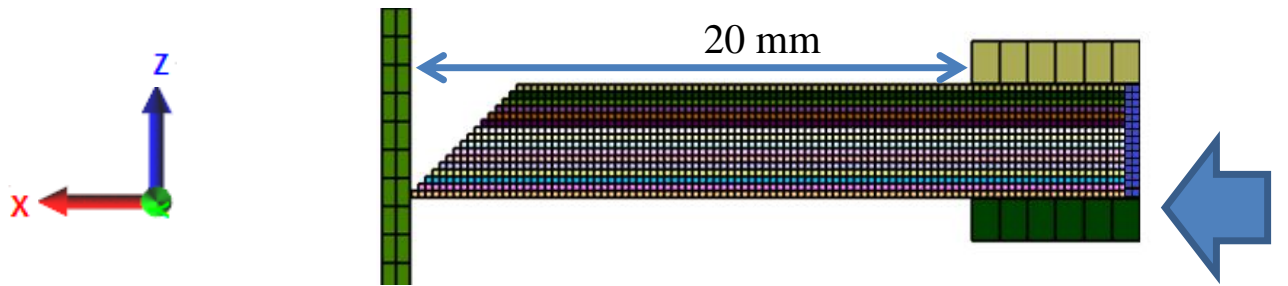

initial velocity of $9 \mathrm{~m} / \mathrm{s}$

Figure 5: One element thick finite element model for 2D simulation

The heavy carriage is non deformable and perfectly tied to the composite structure's end opposite to the contact extremity. This system has an initial velocity of $9 \mathrm{~m} / \mathrm{s}$. The vertical guides are rigid and fixed in space. Penalty contacts are defined between the composite structure and the rigid guides, and between the structure and the base (with a friction of $15 \%$ 
with the ground). Faces of elements perpendicular to the (y) direction are constrained with kinematic symmetry conditions to obtain a 2D plane strain model.

\subsection{Effects of damage modelling on the force}

Typical force-displacement curves obtained in experiments (grey plain and dashed lines) are compared on Figure 6 with the numerical result (orange line) of a previous model with the scale shape mesh at the extremity [8]. The contact force is derived from the total amount of internal energy in the simulation. Numerical and experimental frequencies higher than $1500 \mathrm{~Hz}$ are filtered (moving average). The displacement is that of the heavy carriage centre of mass.

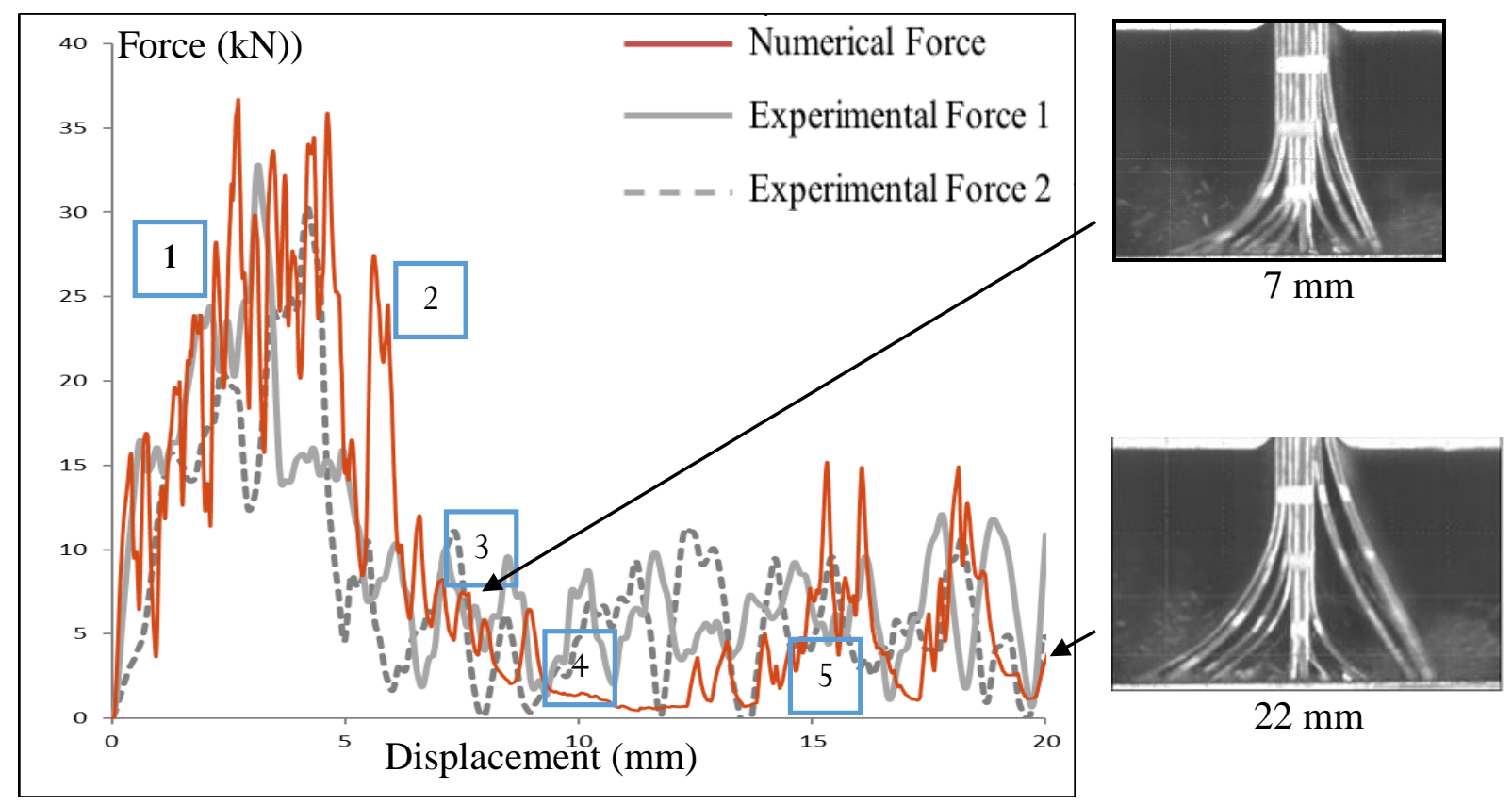

Figure 6: Numerical force - displacement curve versus experiments [8]

As can be seen on Figure 6, the two experimental curves have similar peak force around $32 \mathrm{kN}$. They illustrate the variability in the experiments, but still the global phases in the crushing process remain. We have selected five phases in the force-displacement curve $[8,16-$ 17]. Phase 1 corresponds to the initiation of the crushing from the first contact $(t=0)$ to the peak of force. Oscillations in this phase correspond to the propagation of the acoustic wave generated by the contact, up and down to in the height $(160 \mathrm{~mm})$. The height of the plate damaged during phase 1 corresponds to about one time the thickness for local fragmentation, and three times the thickness for diffuse core damage. Phases 2 and 3 correspond to the global breakdown of the structure. In these phases, the height of the plate that is damaged is the full free height, essentially in splaying (delamination of outer plies). Phase 3 is a transition phase during which the global damage and the local fragmentation modes interact to set the steady process in. Phases 4 to 5 illustrate the beginning of the stabilization process for which the mean crushing force is reached, and its shape as an oscillatory function in time is set.

In a previous work [8], it was demonstrated that the proposed simulation strategy that represents in a different way the local fragmentation (dust) and the global core damage (diffuse) allows to catch the global behaviour in phases 13 and 5. This strategy uses erosion technique to represent the elimination of matter a yield of volume reduction [26]. For the present study, 
element are eroded when they reach $8 \%$ of longitudinal crushing for $0^{\circ}$ plies, and $10 \%$ for $90^{\circ}$ plies. Because of this matter sudden suppression, the model is not precise enough to represent in one hand the peak force because of local instabilities at the vicinity of the ground, and in another hand phase 3-4 of stabilization because of losses of contact when a group of elements were eroded or when a group of bended plies slide on the ground. For that purpose, two phases are particularly examined here, phase 1 and phase 3-4 and the effect of interlaminar fracture toughness on the force and the crushing modes in these phases and in phase 5.

\section{STABILIZATION OF THE CRUSHING PROCESS}

Following the observation done by Lavoie [21], it is aimed here to investigate the effect on the different phases of the crushing process illustrated on figure 6 , and the effect of the interlaminar toughness on the interaction between stress localization in the core of the laminate and the global breakdown and delamination. These interactions are suspected to be at the origin of the zero forces obtained in the simulations because of sudden erosion of groups of elements or loss of contact due to sudden sliding of elements done up to now with the finite elements models that do not use particles [8].

A simulation plan is defined and investigated, using different values of the interlaminar fracture toughness. The maximum normal and tangential stresses are kept constant (except one data set), and energy release rates are modified (see Table 1).

\begin{tabular}{c|llll|l}
\hline Case \# & S (GPa) & T $(\mathrm{GPa})$ & GI $(\mathrm{N} / \mathrm{mm})$ & GII $(\mathrm{N} / \mathrm{mm})$ & Comments \\
\hline 1 & 0.075 & 0.15 & 0.6 & 2.1 & T800s/M21e [8] \\
2 & & & 0.54 & 1.89 & T700s/M21e [16] \\
3 & & & 0.54 & 2.1 & Mixture of \#1 and \#2 \\
4 & & & 0.6 & 1.89 & Mixture of \#2 and \#1 \\
5 & & & 0.6 & 1.6 & GII*75\% for dynamic effects \\
6 & & & 0.75 & 2.1 & GI*1.25\% for dynamic effects \\
7 & & & 0.75 & 1.6 & GI*1.25\% and GII*75\% \\
8 & & & 0.545 & 1.387 & T700s/M21e [24] \\
9 & 0.05 & 0.09 & 0.75 & 1.2 & T800s/M21e [25] \\
\hline
\end{tabular}

Table 1: Example of the construction of one table.

All force-displacements curves obtained from the numerical simulations of cases \#1 to \#9 are compared with experimental force 1 (grey line) presented in Figure 6. Numerical curves obtained for a thickness of $0.25 \mathrm{~mm}$ in the frame of a 2D plane strain hypothesis are scaled in order to be compared with experimental curves obtained on the complete structure of $60 \mathrm{~mm}$ thick. Cases \#1 and \#2 correspond to data sets of different materials obtained from experimental characterization in the laboratory. Cases \#3 and \#4 are combinations of GI and GII from \#1 and \#2. Cases \#5 \#6 and \#7 are adjustments of the values of GI and GII for dynamic effects, that give values not far from data sets of cases \#8 and \#9. Cases \#8 and \#9 are obtained from characterizations tests done in the laboratory, and \#9 was the set used for the model that used the FE-to-particle switch technique [25]. Different comparisons are made to evaluate: the effect of GI value at the fixed values of GII for the T700 and the T800 composites; the effect of GII at the fixed values of GI for the T700 and the T800 composites. Discussions are proposed on the role of the stabilization in the initiation phase 1 on the transition phase 3 and the steady compression process in phases 4-5. 


\subsection{Effect of extreme of GII at different values of GI}

Figure 7, Figure 8, Figure 12, and Figure 12 present the effect of different values of GI at fixed values of GII, on the force-displacement curve. From the comparison of all these curves, it can be noticed that data sets of \#8 and \#9 for GII around $1.3 \mathrm{~N} / \mathrm{mm}$ gives really good correlations of the numerical curves compared to experiment 1, both for the peak force and for the amplitude of the mean force in phases 4-5. But as reminded earlier, these data set exhibit long zones of almost zero force. For \#8, this is due to breakdown of groups of plies that create very big fragments in an unsymmetrical mode of crushing while one half of the structure remains in contact. For \#9, this is due to erosion of elements that are in contact with the ground, , or sliding or groups of elements associated with splaying of outer plies.

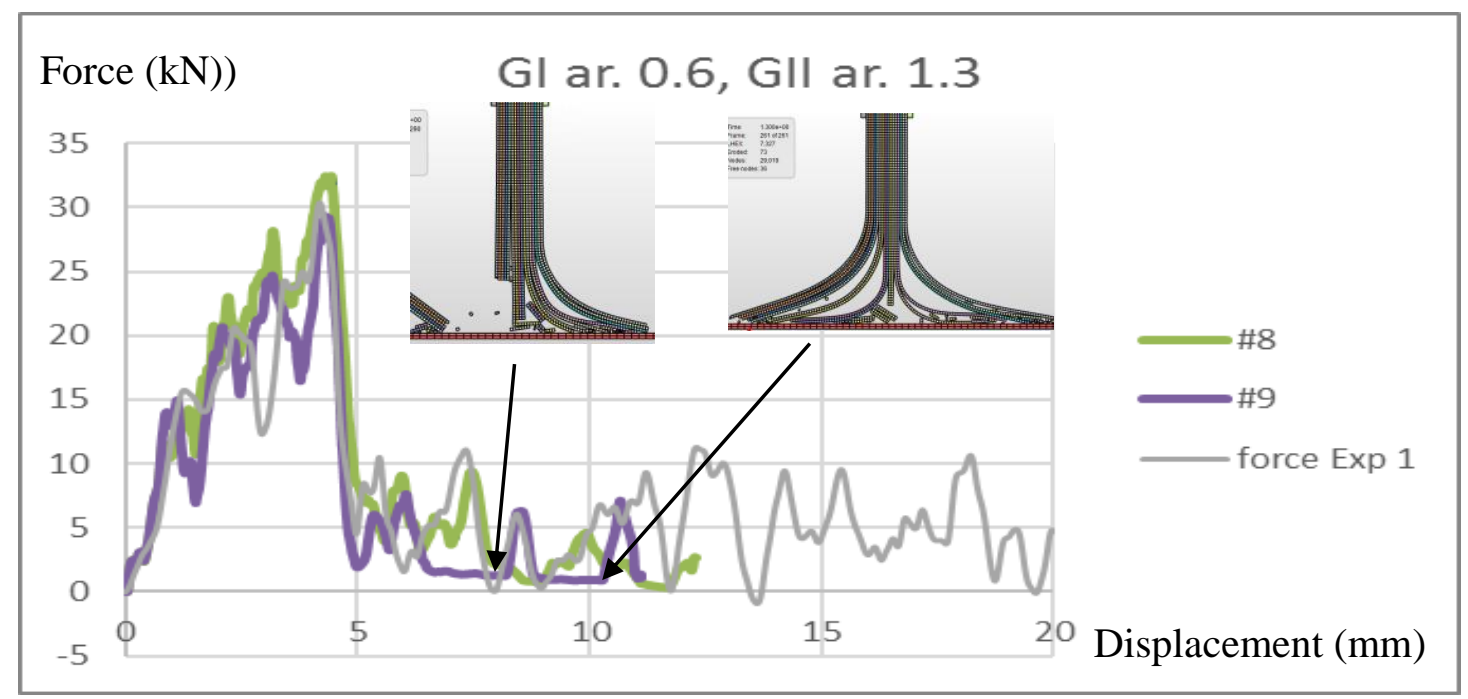

Figure 7: Numerical force - displacement curve at fixed GII around 1.3N/mm versus experiment 1

Comparing the three figures, we can see that increasing GII has two effects. In the initiation phase 1, the force exhibits more oscillations, as observed in the experiment. For and more for \#6 the force-displacement curve is very similar to the experiment up to $8 \mathrm{~mm}$ (phase 3 ).

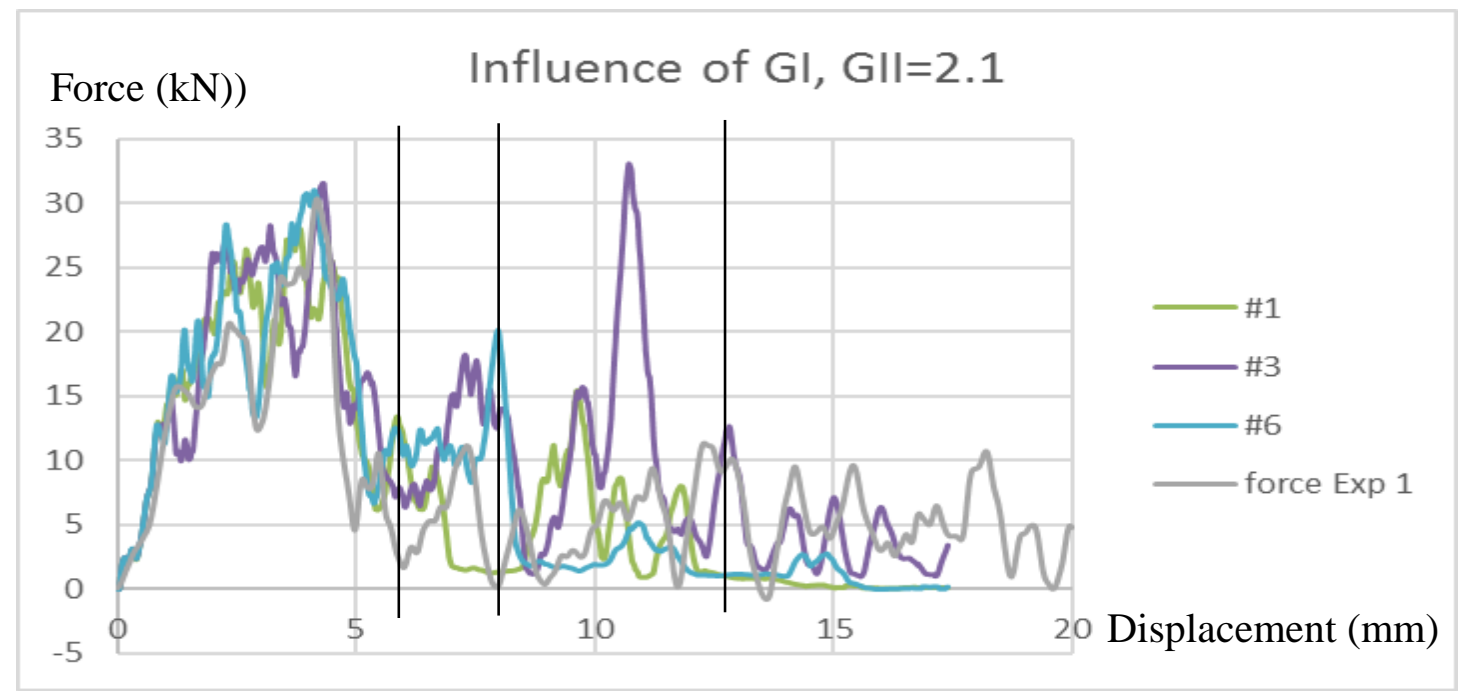

Figure 8: Numerical force - displacement curve at fixed GII=2.1N/mm versus experiment 1 
But for phases 4-5, comparing the curves of Figure 8, we can see that for the same high value of GII, the higher GI the earlier zero forces arise. For \#3, where GI has the lowest value, the frequency and amplitude of the force-displacement curve are very similar to the experimental one in phase $5(>12 \mathrm{~mm})$.

Crushing profiles of \#3, \#1, and \#6, are presented at three stages of displacement, $6 \mathrm{~mm}$, $8 \mathrm{~mm}$, and $12.7 \mathrm{~mm}$ (see Figure 8). The high values of force in phase 5 of case \#3 is explained by the accumulation of debris and the angle of contact between the outer plies and the ground. The angle of contact is explained by debris accumulation between groups of two plies at most (top right in Figure 9). The angle of contact is also quite high which explains the high resistance of \#6 in phase 3 (8mm).

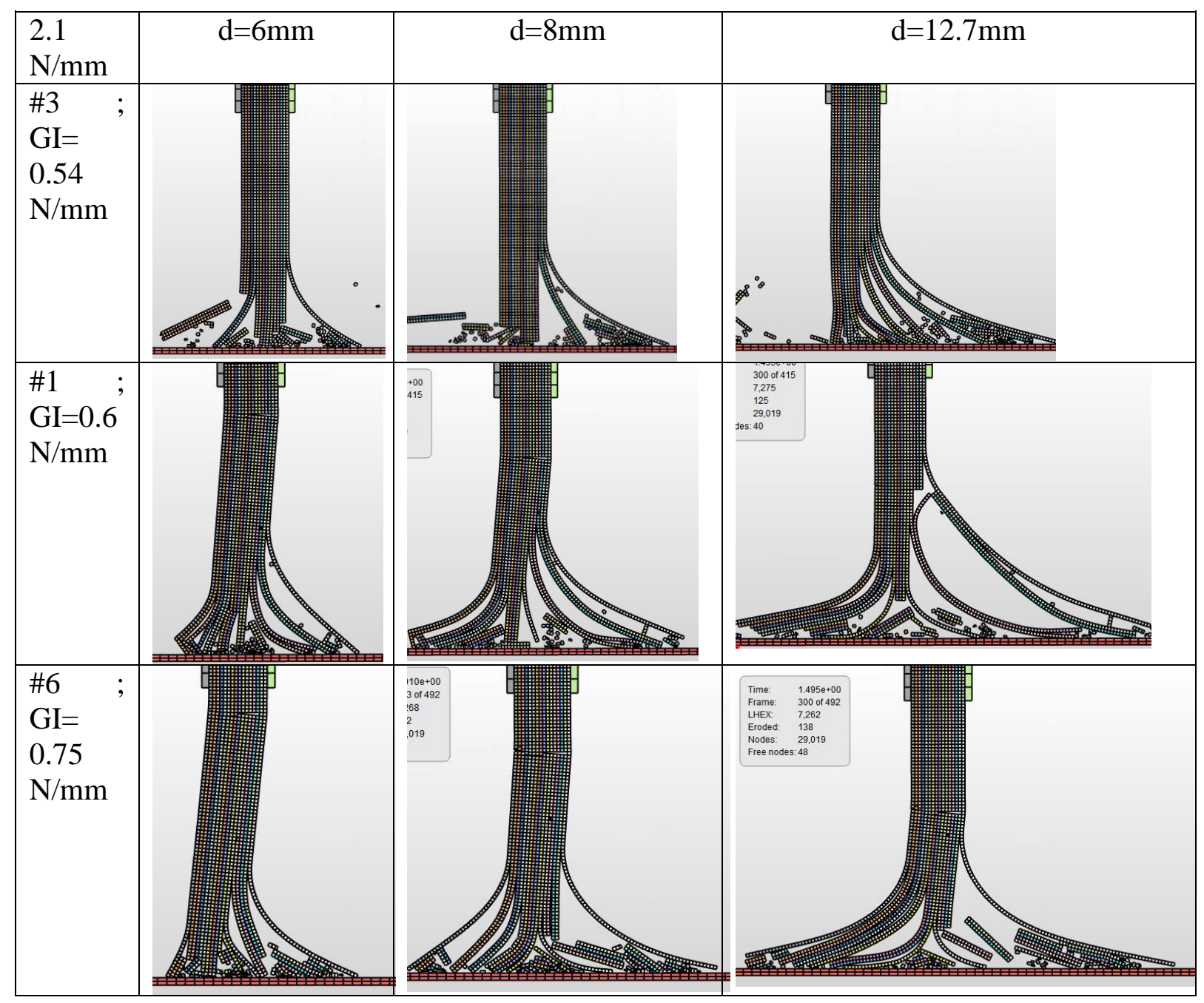

Figure 9: Crushing modes for GII=2.1N/mm for \#3, \#1, and \#6

A good compromise for the force curve is obtained for \#1 for which the outer plies are bended and slide on each other and on the ground with help of debris on which they 'roll' on the left part. On the right part very big debris ensure a non-zero effort for a long period. The angle of contact of the plies on the ground are nevertheless not well reproduced. This explains why the contact force decrease. For \#3, the low value of GI coupled with a high value of GII is responsible of a rapid breakage of groups of plies subjected to bending. Debris are created by the brittle fracture of plies which explains the high amplitude of oscillations of the re- 
sistance force in phase 4 . Even if the mean force is very similar to the experimental one, the crushing process is too brittle and not representative of the tests.

\subsection{Effect of relative variations of GII and GI}

As for GII=2.1N/mm on Figure 8, it can be observed on Figure 10 and Figure 12 that a lower value of GI generates higher amplitudes of oscillations in phase 1, more comparable to the experimental curve. Furthermore, a higher value of GI generates and more losses of contact in phase 3. But on the crushing modes, a higher value of GI helps to stabilize the process and the symmetry of energy dissipation (Figure 11).

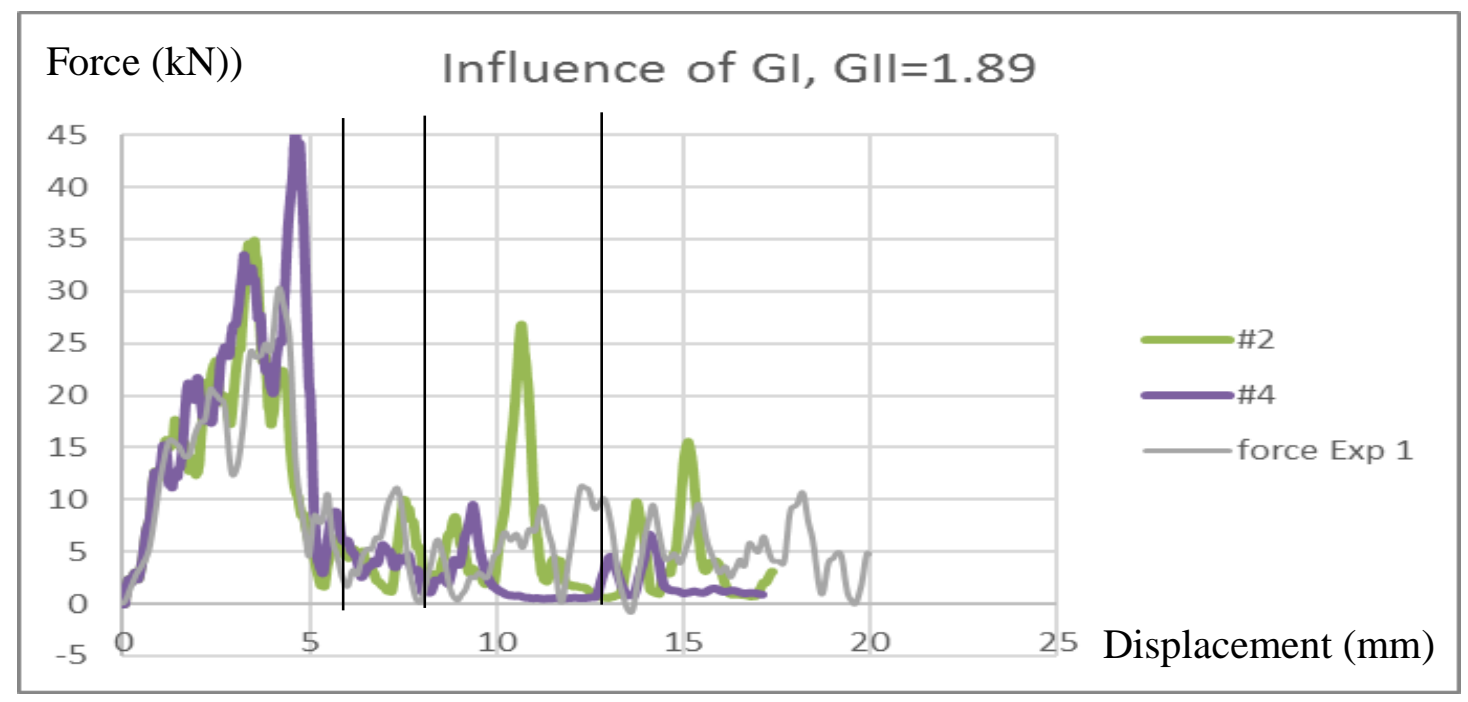

Figure 10: Numerical force - displacement curve at fixed GII $=1.89 \mathrm{~N} / \mathrm{mm}$ versus experiment 1

\begin{tabular}{|c|c|c|c|}
\hline $\begin{array}{l}1.89 \\
\mathrm{~N} / \mathrm{mm}\end{array}$ & $\mathrm{d}=6 \mathrm{~mm}$ & $\mathrm{~d}=8 \mathrm{~mm}$ & $\mathrm{~d}=12.7 \mathrm{~mm}$ \\
\hline $\begin{array}{l}\# 2 ; \\
\mathrm{GI}= \\
0.54 \\
\mathrm{~N} / \mathrm{mm}\end{array}$ & 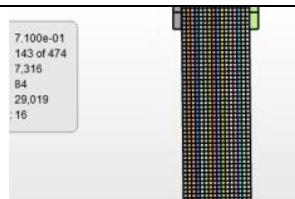 & 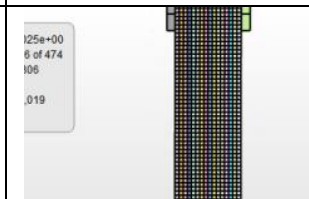 & 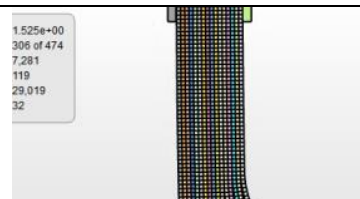 \\
\hline $\begin{array}{l}\# 4 ; \\
\mathrm{GI}= \\
0.6 \\
\mathrm{~N} / \mathrm{mm}\end{array}$ & & & 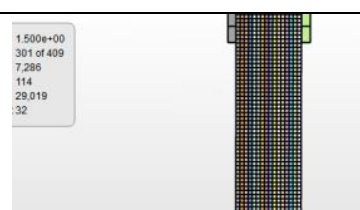 \\
\hline
\end{tabular}

Figure 11: Crushing modes for GII=1.89N/mm for \#2, and \#4

For \#2, which have the same GI $=0.54 \mathrm{~N} / \mathrm{mm}$ than $\# 3$, an artificial peak force appears in phase 4 as well. This peak force is due to the bad configuration of the crushing process (big 
fragments broken by bending. It does not appear in \#8. It is concluded that the peak appears only for higher values of GII in that transition phase. Drop down of the force in phase 3 is realistic for both cases \#5 and \#7.

The crushing profile of cases \#2 and \#3 are very similar. Case \#2 generates two sizes of fragments. Clouds of single elements are confined between outer plies on the right part as in \#3. This causes as in \#3 an angle of contact that is responsible of a non-zero force during almost all the process except during short periods. Peaks of axial force are due to the breakdown of bigger fragments of about $3.5 \mathrm{~mm}$ length and $1.5 \mathrm{~mm}$ width. Should these fragments not completely break but have a smoother behavior, case \#2 would be very close to the test.

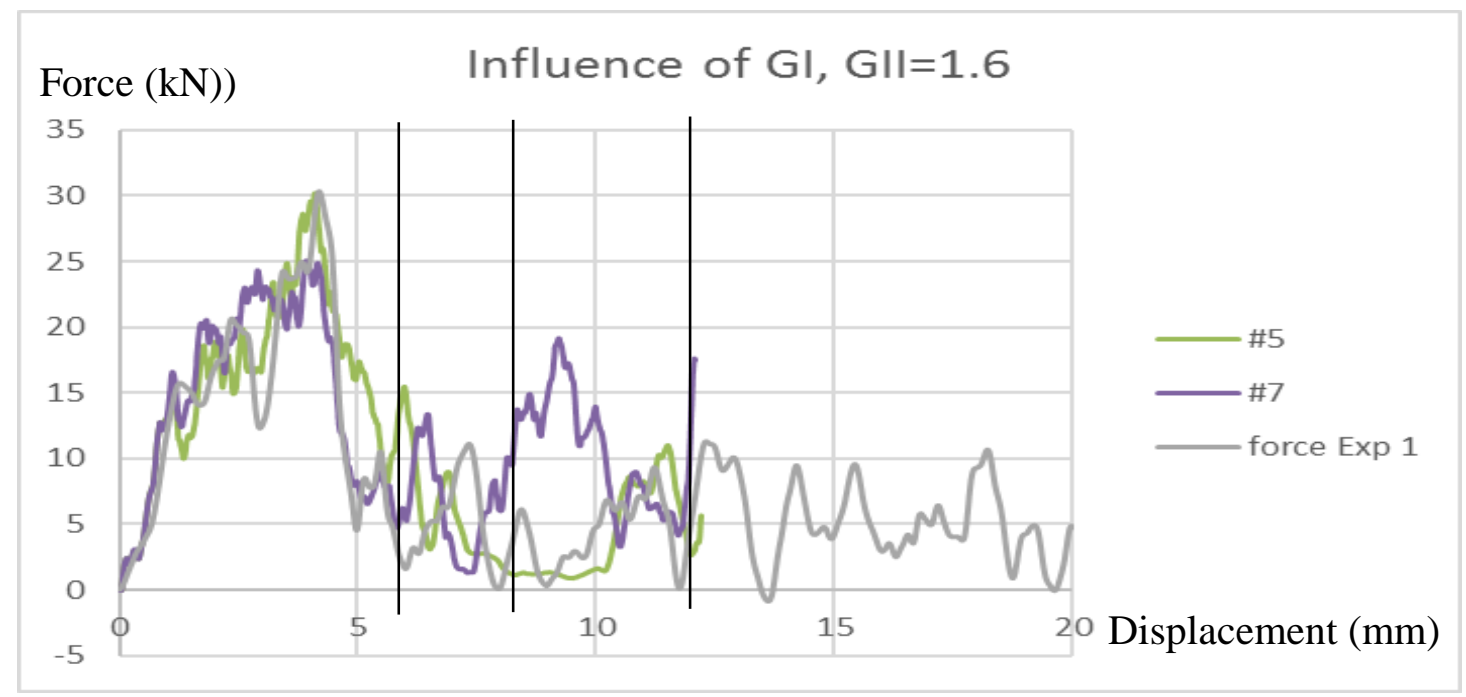

Figure 12: Numerical force - displacement curve at fixed GII=1.6N/mm versus experiment 1

\begin{tabular}{|c|c|c|c|}
\hline $\begin{array}{l}1.6 \\
\mathrm{~N} / \mathrm{mm}\end{array}$ & $\mathrm{d}=6 \mathrm{~mm}$ & $\mathrm{~d}=8 \mathrm{~mm}$ & $\mathrm{~d}=12 \mathrm{~mm}$ \\
\hline $\begin{array}{l}\# 5 \\
\mathrm{GI}= \\
0.6 \\
\mathrm{~N} / \mathrm{mm}\end{array}$ & & & 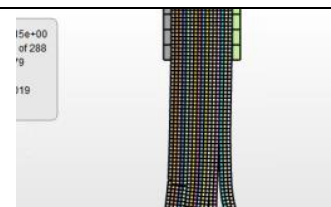 \\
\hline $\begin{array}{l}\# 7 \\
\text { GI }= \\
0.75 \\
\text { N/mm }\end{array}$ & 車 & & 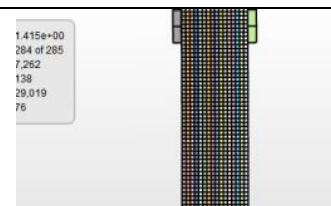 \\
\hline
\end{tabular}

Figure 13: Crushing modes for GII=1.6N/mm for \#5, and \#7 
The same analysis can be conducted for \#5 and \#7. Phase 1 of \#5 is very close to the experiment. Oscillations are higher than for GII $=1.3 \mathrm{~N} / \mathrm{mm}$, but smaller than GII $=2.1 \mathrm{~N} / \mathrm{mm}$. This lead to the general conclusion that a high value of GII is responsible of the amplitude of oscillations of the force-displacement curves, and of the size distribution of the fragments.

This observation is also comforted by the observation of the crushing modes of Figure 13. From the comparison of cases \#6 and \#7, it can also be noticed that a higher GI helps the local fragmentation at the vicinity with the ground, and thus the symmetry of the process.

\subsection{Comparison with experiments}

Figure 14 below shows the evolution of the crushing profile in the different phases of the experiment, same phases used here to analyze the different cases of simulation.

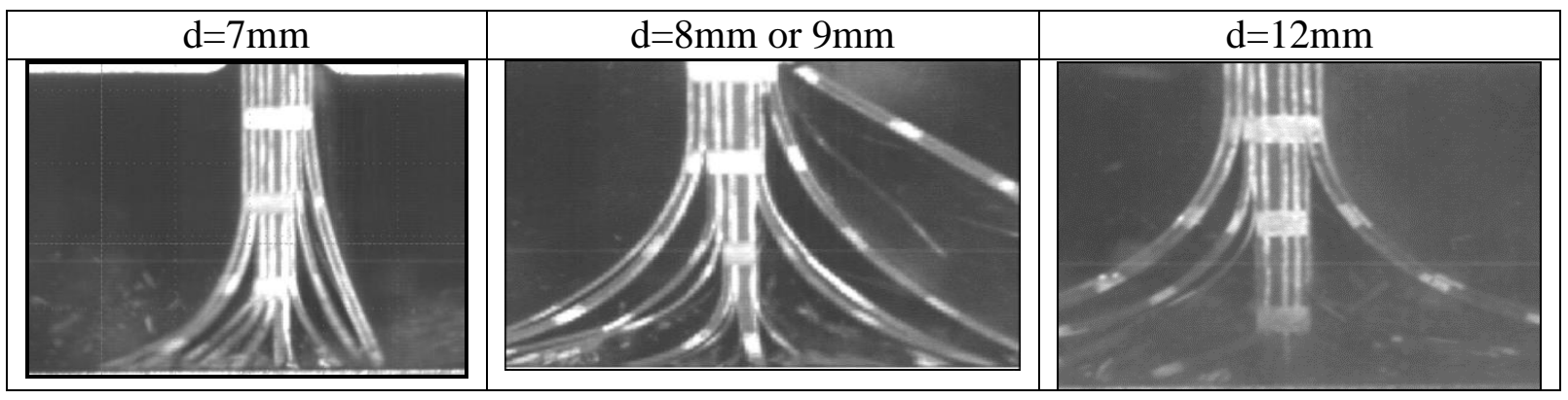

Figure 14: Crushing modes in the experiment

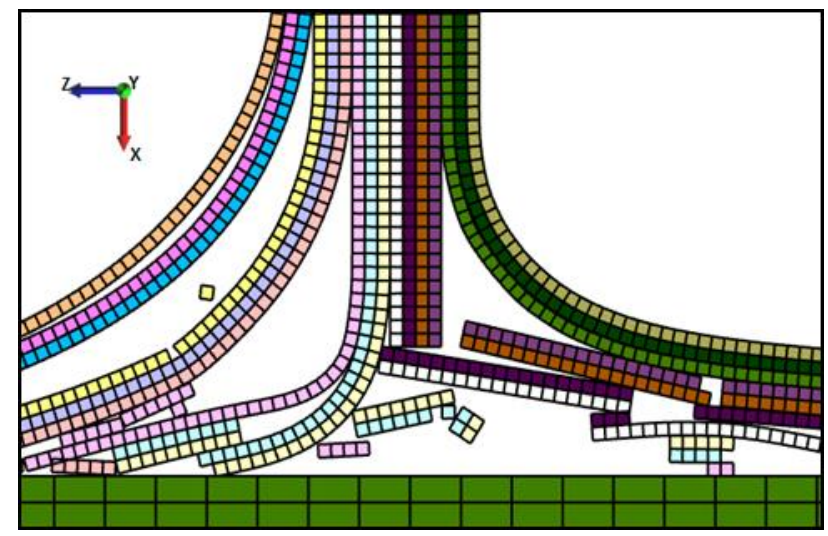

Figure 15: Crushing modes in the previous model : loss of contact due to breakdown of group of elements, erosion, or sliding at a flat angle

Cases \#5 and \#7 give the best comparison with the experiment for the crushing force, as well for the crushing process. Values of GI and GII are quite close from values of \#8 and \#9. GII has a lower value allowing the stabilization of the process. In \#5, the lower value of GI is responsible of the decrease in the resistance force, as it is in the real test. The final decrease in phase 3 is due to creation of big fragments which are not suspected to come from the GII effect, but rather from the erosion technique. Indeed, erosion creates unstable breakdown. It is necessary for us to improve the diffuse damage model in order to stabilize the crushing inside plies, and the formation of kink bands or shear local resistance instead of deleting the matter. 


\section{DISCUSSION AND CONCLUSIONS}

The proposed approach is an enhancement of previous developed numerical models that proved that it is necessary to represent the subtle coupling between the ruin modes of the material and the geometric versus the continuous representation of them to capture the initiation phase of the crushing process and the energy absorption characteristics of the structure. The first model showed that the representation of each size of fragment was necessary and this was done by a FE-particle switching. But this first model also suffered from instabilities at the contact vicinity between the composite sample and the ground. To reproduce in a stable manner, the peak and plateau forces at the macro scale, and the different fragmentation processes at the meso scale, it was necessary to deactivate the local crushing ruin mode in axial compression in the stress-strain material model and to deactivate criteria that were responsible of spurious interactions between out of plane continuous damage inside plies and the cohesive failure between the plies. The present study has proposed an enhancement of this previous model through the introduction of a continuous damage law dedicated to the numerical representation of the highly complex local fragmentation process at the vicinity of the crushing process on the ground in a soft manner. Since oscillations and sudden drops still appear during the crushing process, it was suspected that the interlaminar fracture toughness was not correctly reproduced, leading to an early breakdown of groups of plies because of a dynamic unsteady bending of plies.

The study presented here has investigated the influence of the interlaminar fracture toughness on two measurements, the force-displacement curve, and the crushing process. The different combinations of GI and GII give contrary results for our models, whether the forcedisplacement or the crushing process is analyzed, and two cases could be selected as the best compromise. It has been concluded from the comparison with the experiments that the interlaminar values are not solely responsible of the remaining instabilities of the model. The erosion technique is suspected to be at the origin of the breakdown of groups of plies and the creation of very big fragments in the best configurations of GI and GII. This remain a perspective of our work to enhance the numerical representation of fractures and ruptures.

In a more general analysis, it has been shown that high values of GII are responsible of gig fragments generation, and can either cause the complete rupture of the laminate near the rigid guides. GII is responsible also and for the same reason of the high amplitude of oscillations and disturbing peaks on the resistance force in the force-displacement curve during all the process. This result is concordant with results of the literature that have used other couples of matrix and fibers. For a given value of GII, it has been shown that the higher the GI, the higher also the amplitudes of oscillations in the initiation phase 1, leading also to a more symmetrical crushing mode from the stabilization phase 3 to the steady phase 5 . This stabilization of the crushing process is a good thing up to a certain proportion indeed, because if the stable splaying mode is favored, plies only separate through delamination and slide on each other 'rolling' on debris that are confined between them and the ground, and making a flat angle of contact with the ground. This rapidly leads to decreasing a lot the absorbed energy down to a quasi-null energy dissipation. Before this inefficient splaying mode appears, it has been shown that a right choice of GII and GI combination allows the separation of plies by groups of two or four (for the chosen layup) and at the same time the creation of small to intermediate debris confined between them, forcing the angle of contact and so the bending in the configuration where the accumulated internal energy before rupture is maximized.

It is also shown with these results, that a good design of architecture for a crushing structure is certainly a structure that is not uniform in the height, so that crushing modes are activated at different phases during the process. It is suggested here that the configuration of \#6 
should be used for the initiation phase because it is a good way to release a higher amount of energy and to initiate a stable crushing, then the structure should be designed to have a higher GI compared to GII for outer plies (like \#8, \#9, \#5 or \#7) in order to create small to intermediate debris confined between adjacent plies, forcing them to keep an optimized angle of contact with the ground and help the steady process in a higher level of energy absorption.

\section{ACKNOWLEDGEMENTS}

This work has been funded by the French ANR Program, and led in the frame of the ANR VULOMP2 project. Jerôme Limido of Impetus Afea is sincerely thanked for his great help in user's material modelling and development, and general use of Impetus Code. All the technical staffs of ICA and ISAE-SUPAERO is thanked for their help.

\section{REFERENCES}

[1] G.L. Farley, R. Jones, Crushing characteristics of continuous fiber-reinforced composite tubes, Journal of Composite Materials, 26/1, 37-50, 1992.

[2] D. Hull, A unified approach to progressive crushing of fiber-reinforced composite tubes, Composite Science and Technology, 40, 377-421, 1991.

[3] J.F.M. Wiggenraad, A.L.P.J. Michielsen, D. Santoro, F. Lepage, C. Kindervater, F. Beltran, M. Al-Khalil, Finite element methodologies development to simulate the behaviour of composite fuselage structure and correlation with drop test. Air \& Space Europe, 3/3-4, 228-233, 2001.

[4] M.A. McCarthy, J.F.M. Wiggenraad, Numerical investigation of a crash test of a composite helicopter subfloor structure. Composite Structures, 51, 345-359-256, 2001.

[5] P. Qiao, J. Davalos, E.J. Barbero, Design optimization of fiber reinforced plastic composite shapes. Journal of Composite Materials, 32/2, 177-196, 1998.

[6] P. Feraboli, Development of a Corrugated Test Specimen for Composite Materials Energy Absorption. Journal of Composite Materials, 42/3, 229-256, 2008.

[7] D. Guillon, Etude des mécanismes d'absorption d'énergie lors de l'écrasement progressif de structures composites à base de fibre de carbone. Ph D. Thesis, Université de Toulouse-ICA-ISAE, France, 2008.

[8] F. Tostain, C. Espinosa, S. Rivallant, Crushing of aeronautical composite absorbers using canonical and phenomenological ruin criteria. V ECCOMAS Thematic Conference on the Mechanical Response of Composites, Bristol, United Kingdom, September 7-11, 2015.

[9] G. Sun, F. Xu, G. Li, Q. Li, Crashing analysis and multiobjective optimization for thinwalled structures with functionally graded thickness. International Journal of Impact Engineering, 64, 62-74, 2014.

[10] P. Xu, C. Yang, Y. Peng, S. Yao, D. Zhang, B. Li, Crash performance and multiobjective optimization of a gradual energy-absorbing structure for subway vehicles, International Journal of Mechanical Sciences, 107, 1-12, 2016.

[11] X. Zhang, H. Zhang, Z. Wang, Bending collapse of square tubes with variable thickness. International Journal of Mechanical Sciences, 106, 107-116, 2016. 
[12] H. Assaee, J. Rouzegar, M.S. Saeedi Fkher, A. Niknejad, Axial splitting of composite columns with different cross sections, Thin-walled Structures, 99, 109-118, 2016.

[13] M. Morthorst, P. Horst, Crushing of conical composite shells: a numerical analysis of the governing factors, Aerospace Science and Technology, 10, 127-135, 2006.

[14] M.A. Jimenez, A. Miravete, E. Larrodé, D. Revuelta, Effect of trigger geometry on energy absorption incomposite pofiles, Composite Structures, 48, 107-111, 2000.

[15] A.T. Beyene, E.G. Koricho, G. Belingardi, B. Martorana, Design and manufacturing issues in the development of lightweight solution for a vehicle frontal bumper, International Symposium on Dynamic Response and Failure of Composite Materials (DRaF2014), Procedia Engineering, 88, 77-84, 2014.

[16] H. A. Israr, S. Rivallant, C. Bouvet, J.J. Barrau, Finite element simulation of 0/90 CFRP laminated plates subjected to crushing using a free-face-crushing concept. Composites Part A: Applied Science and Manufacturing, 62, 16-25, 2014.

[17] C. Espinosa, F. Lachaud, J. Limido, J.-L. Lacome, A. Bisson, M. Charlotte, Coupling continuous damage and debris fragmentation for energy absorption prediction by CFRP structures during crushing, Computational Particle Mechanics, 2/1, 1-17, 2015.

[18] L. N.S. Chiu, B. G. Falzon, D. Ruan, S. Xu, R. S. Thomson, B. Chen, W. Yan, Crush responses of composite cylinder under quasi-static and dynamic loading, Composite Structures, 131, 90-98, 2015.

[19] F. Tostain, P. G. Ulecia, L. F.r Alfonso, S. Rivallant, C. Espinosa, Crushing of composite plates: an experimental comparison between laminate and interlock behaviour under static and dynamic loads, $I^{\text {st }}$ International Conference on Impact Loading of Structures and Materials (ICILSM 2016), Turin, Italy, May 22-26, 2016.

[20] A. Esnaola, I. Tena, J. Aurrekoetxea, I. Gallego, I. Ulacia, Effect of fibre volume fraction on energy absorption capabilities of E-glass/polyester automotive crash structures, Composite Part B, 85, 1-7, 2016.

[21] J. A. Lavoie, S. Kellas, Dynamic crush tests of energy-absorbing laminated composite plates, Composites Part A, 27A, 467-475, 1996.

[22] T.J. Vogler, S. Kyriakides, Initiation and axial propagation of kik bands in fiber composites, Acta Mater., 45/6, 2443-2454, 1997.

[23] R.A. Eshkoor, A.U. Ude, S.A. Oshkovr, A.B. Sulong, R. Zulkifli, A.K. Ariffin, Failure mechanism of woven natural silk/epoxy rectangular composite tubes under axial quasistatic crushing test using trigger mechanism, International Journal of Impact engineering, 64,53-61, 2014.

[24] M. Ilyas: Damage modeling of carbon epoxy laminated composites submitted to impact loading, PhD thesis, Université de Toulouse- ICA-ISAE, France, 2010.

[25] C. Espinosa, F. Lachaud, J. Limido, J.-L. Lacome, Combining a CDM model and a FEParticle method to analyse the ruin modes of a composite structure during a crash. European Congress on Computational Methods in Applied Sciences and Engineering, (ECCOMAS 2012), Vienna, Austria, September 10-14, 2012.

[26] Material Sciences Corporation (AMT162 of LS-DYNA): http://www.materialssciences.com/dyna.aspx 